\title{
Application of Genetic Algorithms in Graph Theory and Optimization
}

\author{
Qiaoyan Yang, Qinghong Zeng
}

College of Mathematics, Baoshan University, Baoshan Yunnan, 678000, China

Keywords: Graph theory planning model, Optimization, Genetic algorithm, Peak coverage problems, Coding, Application

\begin{abstract}
There will be a lot of NP- complete problems in graph theory and optimization process, as the most important problem in scientific engineering computing, now it is generally used genetic algorithm to solve. Therefore, this article will mainly study the basic theory of genetic algorithms and graph theory, and put forward the optimization algorithm in graph theory vertex cover problem in the specific application.
\end{abstract}

\section{Introduction}

Genetic Algorithm (GA) is a kind of bionic optimization algorithm based on macro-scientific perspectivep, which relies on the principle of evolutionary changes in nature to selection, crossover, mutation and genetic simulated operator, and by mathematical calculations to achieve group operating globally adaptive optimization, and access to the most optimal solution. In modern science, genetic algorithm in graph theory and optimization process is most common.

\section{Genetic algorithm and graph theory}

\section{Genetic algorithm}

Genetic algorithm based on two factors: the probability of natural selection and genetic search. They calculate the target population through the implementation of genetic manipulation to achieve the internal restructuring of individual groups and iterative, and therefore covers the following four main concepts in the genetic algorithm.

\section{Chromosome}

Chromosome is also called individual, it comes from biology concepts. In the genetic algorithm, chromosomes are using "string" to represent, it represents a vector, in a biological species "string" and the vector can be considered equivalent. For example, the vector $\mathrm{X}=\mathrm{x} 1$, x2 ..... xp. These $\mathrm{x} 1$, $\mathrm{x} 2$...... xp and the like are known to be a string of basic unit vector $\mathrm{X}$, also known as a gene (gene), and $\mathrm{p}$ indicates the total number of genes, also called the length of the string, so that the entire chromosome is also known as gene string.

Population

Population represents a collection of individuals. When using genetic algorithms to solve any problems, we should first randomly select a number of individuals to form a complete whole. But for chromosome geometry, the group can only be regarded as a collection of individual whole chromosomes subset.

Selection operator

There will be a high fitness individuals in the population, they will constitute a new Population, select these groups must rely selection operator. It is assumed that if every individual probability of being selected as $\mathrm{p} 1$ (X1), it depends on the individual factor is $\mathrm{X} 1$ on the environment inclusive fitness $\mathrm{f}(\mathrm{X} 1)=\mathrm{f} 1$, therefore based on the selected operator to select the most commonly used method is to fitness ratio method, also known as the roulette wheel selection, it expressed the following formula:

$$
p_{S}=\left(x_{i}\right)=\frac{f_{i}}{f_{1}+f_{2}+\ldots+f_{n}}
$$




\section{Crossover operator}

Crossover can act simultaneously on two gene strings, it is characterized by a particular gene string interchangeable parts, resulting in a new entity, while the cross section of the exchange is completely random selection, so the crossover of genes strings presence probability swap mechanism. For example, there are two genes string $\mathrm{X}=\mathrm{x} 1 \mathrm{x} 2 \ldots \mathrm{xp}, \mathrm{Y}=\mathrm{y} 1 \mathrm{y} 2 \ldots \mathrm{yp}$, use them pi (i) that if $\mathrm{i}$ meet the (l $\leqslant \mathrm{i} \leqslant \mathrm{n}$ ) this condition, you can achieve mutual exchange position probability.

\section{Graph theory and related planning model}

The main purpose is to plan graph theory model, it is capable of practical problems described for the knowledge of graph theory to answer with scoring, is planning a model formula it uses. In real life, graph theory planning model is more common, such as factory production work arrangements, schedules and so on. These issues also have a common feature, that is to be scored with the graph theory problems, are often those who want to optimize NP-C problem solving. Therefore, planning models based on graph theory to solve the problem of optimizing the key point is to set a reasonable and effective problem solving approach, its purpose is to reduce the size of the space to solve optimization to improve local conditions for the global convergence speed and search accuracy, to enhance work efficiency. It can be seen based on the theory and discuss related issues in the problem of genetic algorithm to graph theory programming model is the effectiveness of having a reasonable; it can realize the majority of problem solving and optimization.

\section{Genetic Programming Model for Solving graph theory-based approach}

Using genetic algorithm problems in graph theory and optimization solver is divided into five steps

Step one: first of all to the programming model to optimize the form of graph theory to solve encode compiled, that is, individual coding genetic algorithm, which is to enable the optimal solution and the individual would form a more intuitive transforming relationship.

Step Two: After the coding constraints to consider the full range of the model, and the constraints of the design and construction of the group's total generation algorithm.

Step three: the algorithm constraints and objective function combined with each other in order to adjust and design conforms to the corresponding fitness function problems, so as to ensure the rapid convergence of the genetic algorithm.

Step Four: Select the rational and effective genetic parameters and genetic operators to participate in the planning graph theory model optimization, genetic algorithm to ensure the smooth unfolding while also achieving stability program execution.

Step five: after the end of the program can make the appropriate interpretation and verification of its optimization results [1].

\section{Optimization solutions based on genetic algorithm graph theory vertex cover problem}

\section{Graph theory vertex cover problem Overview}

Vertices Covering Problems(VCP) discusses in graph theory given graph $\mathrm{G}$ in a subset of vertices (set $S$ ), when the vertex $G$ each edge has at least when one endpoint in $S$, and to ensure that there is no cover G S ', reached $\left|S^{\prime}\right|<|S|$, then $S$ can be considered the minimum coverage of G. Vertex cover problem in biological sequence, information retrieval, the assembly line balancing, scheduling problems, and so the field of theory and practice in both are widely used. Taking into account it belongs to the NP- complete problem, so many approximation algorithms, based on genetic algorithm to determine the minimum coverage issues, and to achieve vertex cover problem graph optimization algorithms, it has simple calculation takes time and fewer restrictions Shaoshao Features. Nearby Find method Also, in order for the optimization problem in graph theory, the paper also specifically genetic algorithms and strategies with local amendments combine to form a more targeted, precision Hybrid Cenetic Algorithm(HGA), by the minimum vertex method to solve and optimize the graph covering problem. 


\section{Examples vertex cover problem analysis}

In graph theory given graph $\mathrm{G}$, assuming its vertices and edges, respectively $(\mathrm{V}, \mathrm{E})$, and the formation of an undirected graph, where $\mathrm{V}$ it means a collection of vertices in the graph, and $\mathrm{E}$ indicates the set of edges. In addition, $|\mathrm{V}|$ and $|\mathrm{E}|$ denote the number of vertices and set the figure edges. Vertex cover is to find a subset of $\mathrm{V}$ and satisfy, and $\mathrm{i}$ and $\mathrm{j}$ belong to the subset $\mathrm{S}$, they are to meet the (i, j) E, its mathematical form expressed as:

$$
\min |S|\left(x_{t} \in S\right)
$$

Given vertex cover problem based on the mathematical expression for the constraint:

$$
\sum_{i=1}^{P} \sum_{j=i+1}^{P} \alpha_{i j}\left(1-x_{i}\right)\left(1-x_{j}\right)=0
$$

When the length of vector $x=\left\lfloor x_{1} x_{2} \ldots x_{p}\right\rfloor$ is $p\left(=|V|\right.$, then $\left(a_{i j}\right)_{p x p}$ is the adjacent matrix, so $x_{i}$ shall comply with the following conditions.

$$
x_{i}=\left\{\begin{array}{l}
1, \text { complywith }_{i} \in S \\
0, \text { non }- \text { complywith }_{i} \in S
\end{array}\right.
$$

On the current vertex cover relevant academic field of graph theory problems approximation algorithm mainly for greedy algorithm. It set in accordance with the degree of the vertex unit Figure descending order, from which select them, then added to cover the maximum vertex set $S$ were, and continue to remove the vertices of $\mathrm{G}$ and all edges and vertices of a given graph is connected, until it is completely deleted. Theoretically reach the greedy algorithm graph vertices issue of minimum coverage, such as it is if you choose the vertex A, then its vertex cover optimum result is B / C / D / E / F, A point will not be covered but becomes redundant vertices. And greedy algorithm also can not get rid of coverage and redundancy in a given graph $G$ vertices $A$, so this will select targeted and higher precision hybrid genetic algorithm HGA [2].

\section{Graph Theory vertex cover problem hybrid genetic algorithm optimization design}

Hybrid genetic algorithm in the calculation purpose is to be achieved by selecting the best chromosome, and they crossover, mutation and genetic combination operator and have an effect, and ultimately the formation of a whole new generation of chromosome groups. While the fitness values of chromosomes in terms of a relatively large, after iterative genetic algorithm converges Operators will be classified as to the best chromosome, and the formation of the optimal solution of the problem.

\section{Design and Analysis of fitness function and chromosome representation}

Generally speaking for vertex cover problem in graph theory given graph it is based on natural coding methods $0 / 1$ coding explained. 1 and 0 , respectively, in or out of coverage, the following encoding methods for the natural vertex cover problem of graphs to explain. Suppose there is a subset of the set of $x, x=\{x 1, x 2 \ldots x n\}$, then the possibility that it can cover the vertex $x=\{x 1, x 2 \ldots x n\}=$ $\{10111010000 \ldots 1\}$.

This indicates that a given vertex cover figure is composed by the vertices $1,3,4$, it forms a graphic infeasible. That is the solution to the given graph does not cover all edges. So based on this case, we use the method of penalty function of the genetic algorithm for solving constrained optimization problems infeasible solutions to solve the problem, it is the principle of optimization penalty given by some figure to be infeasible solutions with constraints no problem into a constrained optimization problem, and at the time did not meet the constraints imposed on the appropriate penalty for the fitness function. In the penalty function, the constant can be present as the value of the penalty function, this value is determined according to the degree of fitness function is violated, the greater the degree of violations, the higher the penalty.

For the vertex cover problem, the objective function represents the minimum cost function of the form, it needs to be based on the target mapped condition function becomes the fitness function form, 
and based on the duality theorem to determine the minimum cost function for maximum profit and the profit function equivalents. It will function to convert the minimum problem to the maximum problem under normal circumstances and multiplied with the cost function, and then multiplied by -1 , and always ensure the fitness function value is positive, the following conversion forms:

$$
f\left(x_{1} x_{2} \ldots x_{p}\right)=\left\{\begin{array}{l}
E_{\text {max }}-g\left(x_{1} x_{2} \ldots x_{p}\right), \text { 当 } g\left(x_{1} x_{2} \ldots x_{p}\right)<E_{\text {max }} \\
0, \text { 当 } g\left(x_{1} x_{2} \ldots x_{p}\right)>E_{\text {max }}
\end{array}\right.
$$

Selection of Max there are many ways, which itself can be used as input parameters, which change with the parameter groups varies.

\section{Design and Analysis of Genetic Operators}

First, the genetic algorithm is based on Darwin's theory of natural selection, which in terms of the strength of the size of the selection has a key role, and therefore the initial selection in genetic algorithms; usually choose a small amount, so that you can fully reflect the diversity of space. This article is selected Dejon exclusion genetic algorithm, which can be used to exclude new generation of offspring and replace the old parent chromosome, thereby increasing the diversity of the population. Figure 1 is a sample space crowding genetic algorithm based on the rule of the selection process.

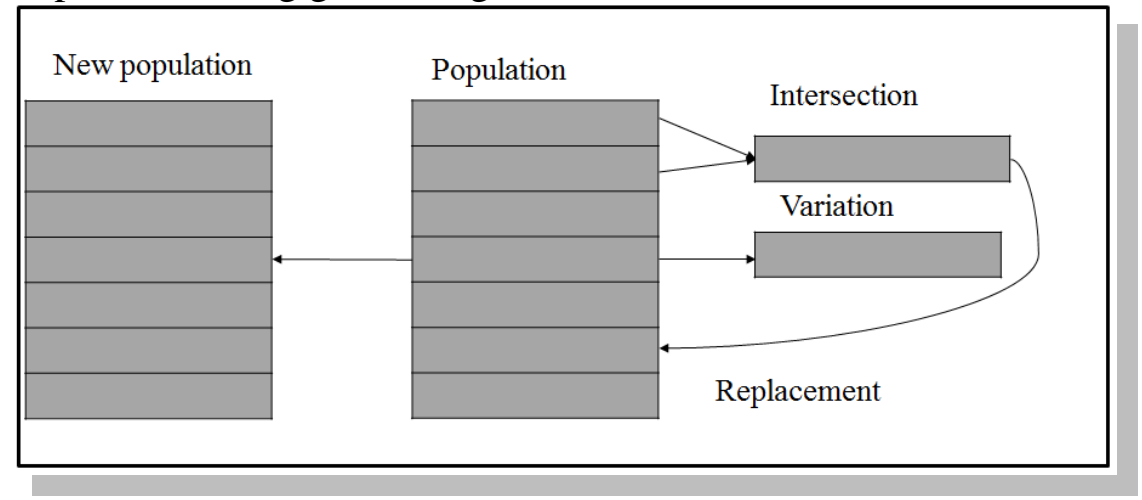

Fig. 1. Based on the sample space selection rules flowchart based on crowed out genetic algorithm

Since the sample space by randomly selecting chromosomes mainly sampling selection is completed, we must first determine the chromosome expectations, and expectations to transform according to the number of offspring. In other words, the basic idea of the algorithm is based on the probability of each chromosome to select and implement adaptation value ratio between the probability assumptions select pi, then it is calculated as follows:

$$
p_{i}=\frac{f_{1}}{\sum_{j=1}^{p o p, \text { size }} f_{j}}
$$

When the probability of chromosome i pi reflected in a certain percentage of the total accounted for chromosome group in fitness, it should calculate the expected value of the chromosome i, namely: ei = pop_size xp, then it should genetic algorithm is described as follows:

$$
\begin{aligned}
& \text { sum } \leftarrow 0 ; \\
& \text { ptr } \leftarrow \operatorname{rand}() ; \\
& \text { fork } \leftarrow 1 \text { to } \quad \text { pop_sizedo }
\end{aligned}
$$




$$
\begin{aligned}
& \text { sum } \leftarrow \text { sum }+e_{k} \\
& \text { while }(\text { sum }>\text { ptr }) d o \\
& \text { Selectchrom }[k] \\
& \text { ptr } \leftarrow \text { ptr }+1 \\
& \text { endwhile } \\
& \text { endfor } \\
& \text { end }
\end{aligned}
$$

According proportional selection method for selecting groups of two chromosomes in the chromosome and chromosome randomly select a point in uniform. Then the switch neutron chromosome string, and generating two offspring chromosomes. Call the scan of chromosome groups - correction mechanism, combined with the resulting offspring chromosomes. Therefore, the final mutation operator should be defined as:

When $X_{i}$ Gene in $X=X_{1} X_{2} \cdots X_{p}$ transforms to $\sim X i$ with probability of $P m$, it represents negation of $X_{i}$, namely: $X_{i} \in\{0,1\}$

\section{Mixed genetic algorithm}

Hybrid genetic algorithm for randomly generated first of length $n$ p chromosome vertex cover problem in a given graph optimization, where p represents the number of vertices in the figure, and the use of standard genetic algorithm, scanning - Fixed and local improvement strategy to improve the chromosome groups. The following is a hybrid genetic algorithm given in Figure G vertex cover problem of optimizing the application process:

$$
\mathrm{t} \leftarrow 0 \text {; }
$$

Initialization population $\mathrm{P}(\mathrm{t})$;

Scan - the amendment process

Evaluation population $\mathrm{P}(\mathrm{t})$;

While (termination condition is not satisfied);

The complete genetic operators acting on population $\mathrm{P}(\mathrm{t})$ generated by the group $\mathrm{C}(\mathrm{t})$; and

A scanning - the revision process;

Implementation of Local-Improvement-Search Process;

Evaluation of group $\mathrm{C}(\mathrm{t})$;

$$
\mathrm{t} \leftarrow \mathrm{t}+1
$$

End

Such as the mixed genetic algorithm derivation, in the output of the vertex cover problem graph optimal coverage optimization, we can achieve the best explanation for the optimization of graph theory [3].

\section{Conclusions}

Based on the genetic algorithm to explore in graph theory and optimization, presents the best solution on the settlement of the minimum vertex cover problem given graph, namely hybrid genetic algorithm, which cover issues for the implementation of the scan - correction algorithm, and the realization of the local optimization techniques to generate new progeny groups, and finally generate the optimal solution. All this shows the superiority of the genetic algorithm, confirming the robustness of its design can be applied to the vertex cover problem and more different topologies of graph theory given graph.

\section{Acknowledgments}

This article is a general project in Yunnan Province Department of Education, Project Name: Study on the Classification 2pq $\wedge 2$ rank 5 degree arc transitive graphs, NO: 2014Y486; Yunnan 
Provincial Department of Education is also the general project, Project Name: K times short-circuit and short-circuit problem algorithm, NO: 2014Y485.

\section{References}

[1] Kong Dejian. Multiobjective optimization problem research based on improved genetic algorithm. Computer simulation,2012,29(2):213-215.

[2] Liu Xiang. The application of Genetic Algorithm and Its graph theory in planning model. The PLA Information Engineering University,2007,11-15.

[3] Hong Hongwei Application of genetic algorithm in graph theory and optimization. Xi'an University of Electronic Science and Technology,2000.22-39. 